Case

Report

\title{
Neurilemmoma Showing a Unique Growth Pattern in the Lateral Chest Wall: Both Inside and Outside the Thoracic Cavity
}

\author{
Sun Jun Ahn, MD, ${ }^{1}$ Kyung Joo Park, MD, ${ }^{1}$ Joo Sung Sun, MD,${ }^{1}$ Ho Choi, MD,${ }^{2}$
} and Yong Hee Lee, $\mathrm{MD}^{3}$

\begin{abstract}
We report the first case of the intercostal neurilemmoma showing a unique growth pattern in the lateral chest wall (both inside and outside the thoracic cavity). Usually, intrathoracic neurilemmoma is found in the posterior mediastinum and bulge from the inner surface of the bony thorax toward the thoracic cavity. This unique growing pattern of neurilemmoma arising from intercostal nerve was clearly demonstrated by computed tomography and ultrasonography.
\end{abstract}

Keywords: neurilemmoma, chest wall, computed tomography

\section{Introduction}

Neurilemmoma (schwannoma) is a well-encapsulated benign tumor that originates from Schwann cells. Prognosis is excellent, and recurrence of the tumor is rarely reported after surgical resection. ${ }^{1)}$ Intrathoracic neurilemmoma is usually found in the posterior mediastinum and bulge from the inner surface of the bony thorax toward the thoracic cavity. ${ }^{2,3)}$ Extrathoracic neurilemmoma is extremely rare, and Sakurai, et al. reported one case of extrathoracic neurilemmoma mimicking a subcutaneous tumor of the lateral chest wall. ${ }^{4}$ ) To the best of our knowledge, we report the first case of dumbbellshaped neurilemmoma that had grown both outside and inside the thoracic cavity in the lateral chest wall.

${ }^{1}$ Department of Radiology, Ajou University School of Medicine, Suwon, Korea

${ }^{2}$ Department of Thoracic and Cardiovascular Surgery, Ajou University School of Medicine, Suwon, Korea

${ }^{3}$ Department of Pathology, Ajou University School of Medicine, Suwon, Korea

Received: August 29, 2011; Accepted: November 2, 2012

Corresponding author: Joo Sung Sun, MD. Department of Radiology, Ajou University School of Medicine, San 5, Wonchon-dong, Yeongtong-gu, Suwon 443-721, Korea

Email: sunnahn@ajou.ac.kr

(C)2014 The Editorial Committee of Annals of Thoracic and Cardiovascular Surgery. All rights reserved.

\section{Case Report}

A 57-year-old woman with a palpable right lateral chest wall mass visited our hospital. She recognized the mass three weeks before this first visit. She was a nonsmoker and had no other disease history. There was no tenderness or pain of the chest wall mass on physical examinations. All laboratory tests were within normal limits. A plain chest radiograph demonstrated a bulging soft tissue shadow of the right lower lateral thorax without adjacent rib destruction. Coronal section images of a computed tomographic (CT) scan depicted a welldefined dumbbell-shaped mass with internal heterogeneous enhancement in the right lower lateral chest wall. The mass was located along the right 9th intercostal space and bulged toward both the extrathoracic and intrathoracic cavity and was compressing the liver surface. Additionally, erosion of the right 9th rib inferior margin was suggested (Fig. 1). Axial CT showed mild rim enhancement with some enhancement in the central portion on post-contrast CT (Fig. 2). The tumor was suspected to be a neurogenic tumor originating from an intercostal nerve, but its growth pattern was unfamiliar, so the tentative diagnosis included neurogenic tumor, desmoid tumor, and sarcoma of the chest wall. Therefore, ultrasonography-guided core needle biopsy was performed for pathologic diagnosis. Ultrasonography revealed the 


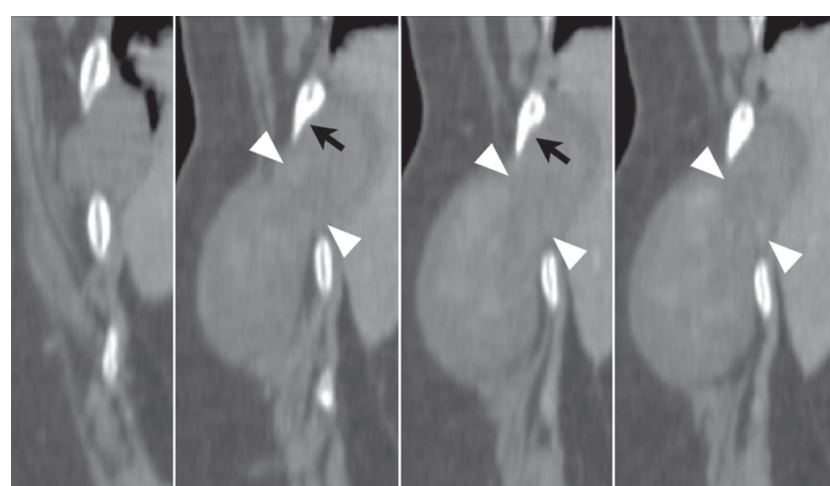

Fig. 1 Coronal section images of CT clearly demonstrating a dumbbell-shaped mass lying from the inner chest wall to the outer chest wall through the 9th intercostal space (white arrowheads). Erosion of the 9th rib inferior margin is also depicted (black arrows).

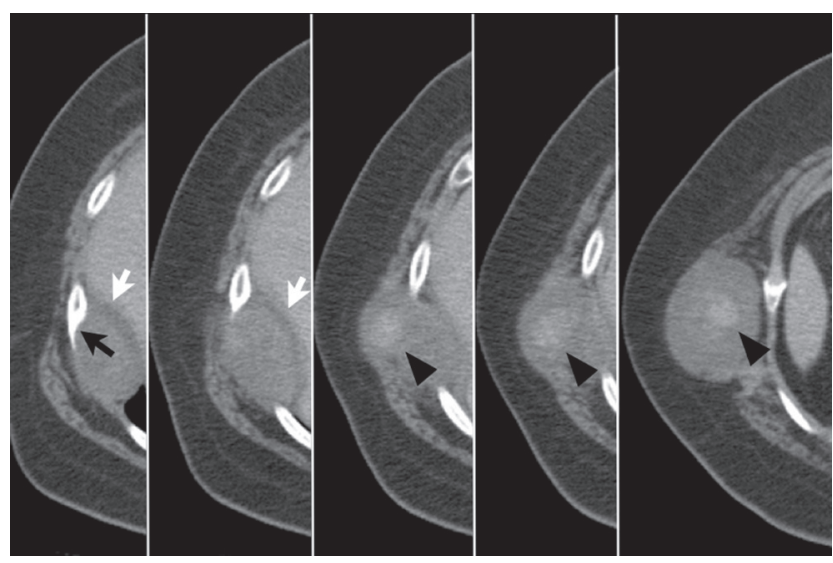

Fig. 2 Axial post-contrast CT revealing the inner component of the mass compressing the liver (white arrows) and bulges through the intercostal space into the outer thoracic wall. The tumor erodes the adjacent inferior margin of the 9th rib (black arrow) and shows central enhancement (black arrowheads).

tumor showing a hypoechoic rim with a central hyperechoic appearance with dumbbell shape. The tumor lay from the inner chest wall to the outer thoracic cavity (Fig. 3).

The patient underwent tumor excision. Under general anesthesia, a $7-\mathrm{cm}$ transverse incision was created along the right 9 th intercostal space to expose the tumor. After the tumor had been exposed, it was relatively easy to separate it from the muscle, rib, and parietal pleura. Grossly, the tumor was a dumbbell-shaped, soft, wellencapsulated, rubbery mass. On the cut section, it had a mostly yellow myxoid appearance with focal hemorrhage. Microscopically, the tumor consisted of central areas that showed a dense spindle cell pattern with

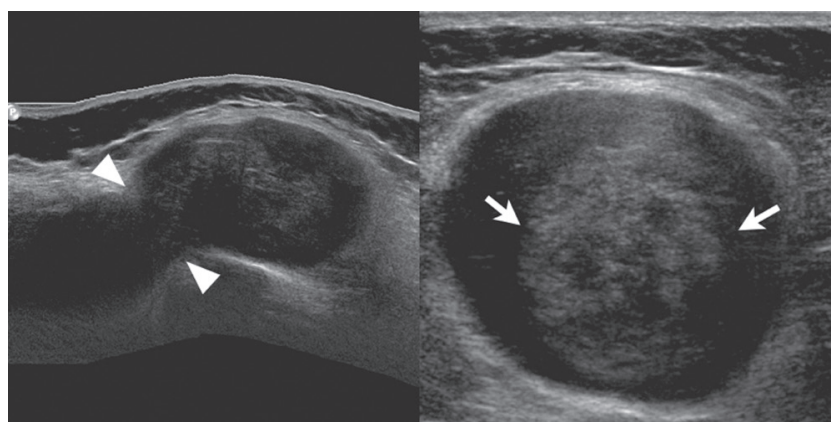

Fig. 3 Ultrasonography also clearly shows a dumbbell-shaped configuration through the intercostal space (arrowheads). An axial scan of ultrasonography demonstrates central high echogenicity (white arrows) and peripheral low echogenicity.

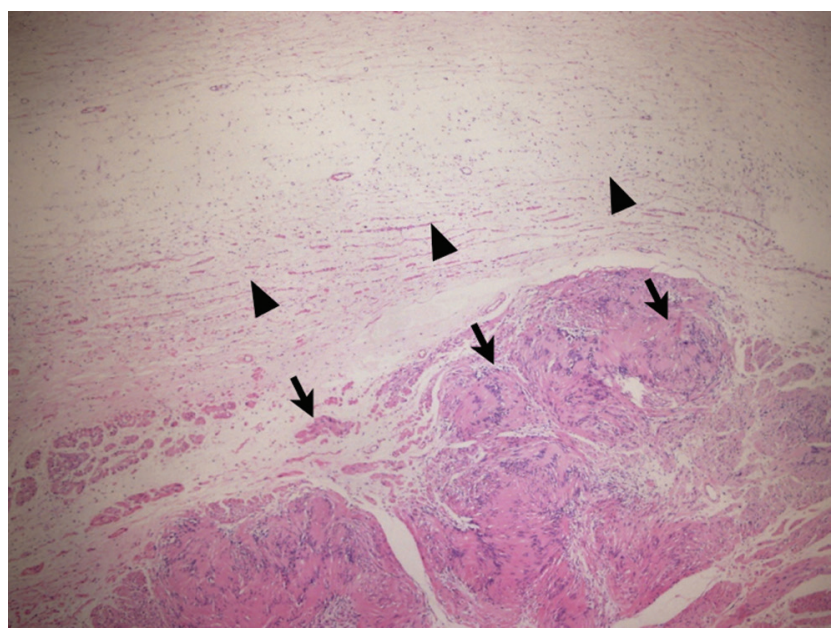

Fig. 4 Histopathologic examination ( $\times 100$ magnification $)$ of the tumor reveals a tumor composed of a central cellular area (Antoni type A tissue) having Verocay bodies and nuclear palisading (arrows) and a peripheral hypocellular area (Antoni type B tissue) (arrowheads).

nuclear palisading and interlacing fascicles, in addition to peripheral areas that were less cellular with myxomatous changes (Fig. 4). Immunohistochemically, the tumor cells were positive for S-100 protein. The final diagnosis was neurilemmoma. She has been followed-up for three months with no evidence of recurrence.

\section{Discussion}

Primary chest wall tumors often originate from neural tissue, and most of them are benign. ${ }^{5,6)}$ Neurogenic tumors are classified as nerve cell origin tumors (i.e., ganglioneuroma, neuroblastoma) or nerve sheath origin tumors (i.e., neurilemmoma, neurofibroma), on the basis 
of cell origin. Most neurilemmomas originate from the posterior mediastinum bulging from paravertebra toward the thoracic cavity; neurilemmomas arising from the intercostal nerve of the lateral chest wall are relatively uncommon, only $5 \%$ of thoracic neurilemmoma. ${ }^{2)}$ Furthermore, only one case exists of an extrathoracic neurilemmoma mimicking a subcutaneous tumor, in the English literature. ${ }^{4)}$ Dumbbell or 'hourglass' neurilemmomas have been reported in several cases ${ }^{7}$; most of these cases were located in paravertebral regions. To the best of our knowledge, no previous report has been published about a case of neurilemmoma growing both inside and outside of the lateral chest wall.

Normally, the intercostal nerve lies between the intracostal and internal intercostal muscles and courses along the subcostal groove with the intercostal vessel. It could be postulated that a neurilemmoma growing toward the outer chest wall might be blocked by the inferior margin of the adjacent rib. Therefore, a neurilemmoma arising from the intercostal nerve of the chest wall usually bulges toward the thoracic cavity. There would be two possible conditions allowing a unique growth pattern of neurilemmoma both inside and outside of the chest wall. The first one is that the neurilemmoma arose from the lateral cutaneous branch of the main intercostal nerve. In the chest wall, the intercostal nerve travels a subcostal course and provides lateral cutaneous branches near the mid-axillary line that travels anteriorly and posteriorly in subcutaneous layers. Considering the anatomy of the intercostal nerve, it is possible that the neurilemmoma in this case arose from the junction of lateral cutaneous branching from the intercostal nerve. The second possibility is anatomic variation. The intercostal nerve might lie downward away from the subcostal groove, blocking the outer growth of neurilemmoma. Unfortunately, in this case, the origin of the tumor could not be clearly investigated, whether the tumor originated from the main intercostal nerve or lateral cutaneous branch of intercostal nerve because the operation was performed through an incisional window.

Generally, chest wall neurilemmomas are evaluated by radiologic examination, including $\mathrm{CT}$ and magnetic resonance imaging (MRI). Particularly, MRI should be performed in patients with suspicious posterior mediastinal neurogenic tumors to exclude intra-spinal tumor extension. When a neurogenic tumor occurs in the poste- rior mediastinum, the typical signal intensity of MRI allows for high accuracy of diagnosis. ${ }^{8)}$ Thus, preoperative radiologic evaluation alone is enough without needle aspiration biopsy. In this case, the growth pattern of the tumor was not usual, since the neurilemmoma arose from the intercostal nerve. However, the tumor was demonstrated as peripheral low echogenicity with central high echogenicity with ultrasonography. Similarly, the tumor showed central enhancement on post-contrast CT. This might correspond to pathologic findings as follows: predominant central hypercellular area (Antoni type A tissue) and predominant peripheral hypocellular area (Antoni type B tissue) with myxomatous change of the tumor.

In conclusion, neurilemmomas arising from the intercostal nerve could grow both inside and outside of the chest wall, and we report the first case of neurilemmoma showing such a unique growth pattern in the lateral chest wall.

\section{Disclosure Statement}

None to disclose.

\section{References}

1) Lee JY, Lee KS, Han J, et al. Spectrum of neurogenic tumors in the thorax: CT and pathologic findings. J Comput Assist Tomogr 1999; 23: 399-406.

2) Davidson KG, Walbaum PR, McCormack RJ. Intrathoracic neural tumours. Thorax 1978; 33: 359-67.

3) Duwe BV, Sterman DH, Musani AI. Tumors of the mediastinum. Chest 2005; 128: 2893-909.

4) Sakurai H, Hada M, Mitsui T, et al. Extrathoracic neurilemoma of the lateral chest wall mimicking a subcutaneous tumor: report of a case. Ann Thorac Cardiovasc Surg 2006; 12: 133-6.

5) Sabanathan S, Salama FD, Morgan WE, et al. Primary chest wall tumors. Ann Thorac Surg 1985; 39: 4-15.

6) Murray RJ, Criner GJ, Siegel E. Multiple schwannomas presenting as a mass of the lateral chest wall. AJR Am J Roentgenol 1988; 151:1250-1.

7) Payer M, Radovanovic I, Jost G. Resection of thoracic dumbbell neurinomas: single postero-lateral approach or combined posterior and transthoracic approach? J Clin Neurosci 2006; 13: 690-3.

8) Sakai F, Sone S, Kiyono K, et al. Intrathoracic neurogenic tumors: MR-pathologic correlation. AJR Am J Roentgenol 1992; 159: 279-83. 